\title{
Inklusionsorientierung im Sozialraum - Verpflichtung und Herausforderung
}

\author{
Clemens Dannenbeck
}

I. Einführung - Der Inklusionsdiskurs

II. Feuilletonistische Randbemerkungen aus dem beschädigten Leben oder (fast) wie im richtigen Leben

1. Die Grenzen der Inklusion in der inklusiven Praxis 50

2. Ramponierte Teilhabe - der vermessene inklusive Sozialraum $\quad 50$

3. Einsicht in die eigene Verstricktheit 51

4. Inklusion einfach machen - und alles wird gut 51

III. Wissenschaftlicher Teil $\quad 51$

$\begin{array}{ll}\text { IV. Ausblick } & 56\end{array}$

\section{Einführung - Der Inklusionsdiskurs}

Im Folgenden wird versucht, die Notwendigkeit der stärkeren Berücksichtigung einer sozialräumlichen Perspektive in der politischen und öffentlichen Inklusionsdebatte zu begründen, um anschließend in Ansätzen herauszuarbeiten, zu welchen behindertenpolitischen Blickverschiebungen eine solche Fokussierung des Sozialraums führen würde. Ausgangspunkt der Argumentation ist folgende Wahrnehmung des Inklusionsdiskurses, wie er sich infolge der Ratifizierung der UN-BRK durch die Bundesrepublik Deutschland seit dem Jahr $2009^{1}$ präsentiert: Die Ratifizierung der UN-BRK führte zu einem (für manche durchaus überraschend) hohem Handlungsdruck und -bedarf auf diversen politischen Entscheidungsebenen, nicht zuletzt durch die mit der freiwilligen Ratifizierung des Fakultativprotokolls verbundenen inhärenten Kontroll- und Qualitätsmechanismen, die die Reaktionen und Umsetzungsprozesse national wie international beglei-

1 Die UN-Konvention über die Rechte von Menschen mit Behinderung ist seit dem 26. März 2009 in der Bundesrepublik Deutschland rechtsverbindlich. Grundlage ist das Gesetz zum Übereinkommen der Vereinten Nationen vom 13. Dezember 2006 über die Rechte von Menschen mit Behinderungen sowie das Fakultativprotokoll vom 13. Dezember 2006 zum Übereinkommen der Vereinten Nationen über die Rechte von Menschen mit Behinderungen (Deutsch, Englisch, Französisch); vgl. hierzu: http://www.institut-fuer-menschenrechte.de/de/monitoring-stelle.html (Stand 10. Juni 2012). 
ten und fortgesetzter öffentlicher Beobachtung und Kritik aussetzen. Folge davon sind Aktionen, Absichtserklärungen und Beteuerungen, die sich zum Teil in verstärkten integrationspolitischen Bemühungen ${ }^{2}$, zum Teil aber auch in einer inflationären Inklusionsrhetorik $^{3}$ niederschlagen. Beispielhaft für die semantische Übernahme bei gleichzeitiger inhaltlicher Aushöhlung der Forderung nach voller selbstbestimmter gesellschaftlicher Teilhabe für alle Menschen ist zum einen die Übersetzungsgeschichte der deutschsprachigen Fassung des Textes der UN-BRK ${ }^{4}$, zum anderen etwa die Umbenennung des so genannten bayerischen Weges der Integration durch eine Vielfalt schulischer Angebote zur Inklusion durch eine Vielfalt schulischer Angebote ${ }^{5}$. Die Konvention ist aber nicht nur Diskursanlass, sondern auch geltendes Recht ${ }^{6}$. In der inflationären Inklusionsrhetorik spiegelt sich der systematische Versuch, von der gesamtgesellschaftlichen Dimension der Herausforderung durch Inklusion abzulenken und den umfassenden Anspruch, der sich aus dem Geist der UN-BRK ableiten lässt, auf eine alleinige Aufgabe bisweilen des Bildungssystems, bisweilen auch nur von Sonderschulen, möglicherweise sogar zur Sache einzelner pädagogischer Lehrkräfte zu reduzieren. Einem solchermaßen politisch verkürzten Verständnis von Inklusion entspricht unverkennbar eine Strategie der Individualisierung des Problems: Inklusion darf, kann und soll doch jeder - ob in Schule oder Betrieb - „einfach machen“7, zumal - so die stets beifalls-

2 Zum Stand der Dinge vgl. den Ersten Staatenbericht der Bundesrepublik Deutschland zum Übereinkommen der Vereinten Nationen über Rechte von Menschen mit Behinderungen, vom Bundeskabinett beschlossen am 3. August 2011, abrufbar unter: http://www.bmas.de/SharedDocs/Downloads /DE/staatenbericht-2011.pdf?_blob=publicationFile (Stand 10. Juni 2012) sowie den Nationalen Aktionsplan der Bundesregierung zur Umsetzung der UN Behindertenrechtskonvention vom August 2011, abrufbar unter: http://www.bmas.de/SharedDocs/Downloads/DE/PDF-Publikationen/a740nationaler-aktionsplan-barrierefrei.pdf?_blob=publicationFile (Stand 10. Juni 2012).

3 Vgl. hierzu den Beitrag von Wocken, Über die Entkernung der Behindertenrechtskonvention. Ein deutsches Trauerspiel in 14 Akten, mit einem Vorspiel und einem Abgesang, Inklusion-online, Zeitschrift für Inklusion Nr. 4 2011, abrufbar unter http://www.inklusion-online.net/index.php/ inklusion/article/view/139/135 (Stand 10. Juni 2012).

$4 \mathrm{Vgl}$. hierzu die Schattenübersetzung der UN-BRK durch Netzwerk Artikel 3: http://www.netzwerkartikel-3.de/index.php?view=article\&id=93:international-schattenuebersetzung （Stand 10. Juni 2012).

5 Vgl. das gleichnamige Konzeptpapier des Bayerischen Staatsministeriums für Unterricht und Kultus vom 1. August 2011 IV.6 - S 8040.5.1 - 4a.107922 zur Umsetzung der UN-Behindertenrechtskonvention in Bayern hinsichtlich des Gesetzentwurfs zur Änderung des Bayerischen Gesetzes über das Erziehungs- und Unterrichtswesen - Umsetzung der UN-Behindertenrechtskonvention im bayerischen Schulwesen (Inklusion) (BT-Drucks. 16/8100 vom 28.03.2011) - dazu siehe auch: http://www.verwaltung.bayern.de/portal/cl/1058/Gesamtliste.html?cl.document=4036315 (Stand 10 . Juni 2012).

6 So die bündige Formulierung von Hirschberg in ihrem Beitrag auf der Fachtagung der Informations- und Beratungsstelle Studium und Behinderung (IBS) des Deutschen Studentenwerks (DSW) am 15. Juni 2012 in Berlin.

7 So das Motto zur Dachkampagne des Bundesministeriums für Arbeit und Soziales zur Umsetzung der UN Behindertenrechtskonvention und Namensgeberin der Konferenz „Inklusion einfach machen - von Bildung über Ausbildung in Arbeit“"vom 7. Dezember 2011. 
trächtige Formel - Inklusion schließlich im Kopf beginnt. In einer derartigen strategischen Ausrichtung werden Ressourcen- und Haltungsaspekte gegeneinander ausgespielt. Im Übrigen ist leider auch zu beobachten, dass die Fachdisziplinen der Integrations- und Inklusionspädagogik im Eifer des Gefechts und angesichts des momentan in der inklusionspolitischen Morgendämmerung verspürten Rückenwinds keineswegs davor gefeit sind, dieses (meiner Ansicht nach verhängnisvolle) Spielchen mitzuspielen: Da schießen so genannte und selbst ernannte „Inklusionsschulen“ wie Pilze aus dem Boden und jeder kleine Integrationsfortschritt wird mittlerweile als Mosaiksteinchen einer sich am Horizont bereits mehr oder weniger deutlich abzeichnenden inklusiven Gesellschaft gefeiert. Der breiten Öffentlichkeit bleibt dabei nur eines, nämlich sich die neue politisch korrekte Leitvokabel anzueignen - was bis gestern Integration hieß, wird fürderhin Inklusion genannt - ansonsten scheinen die besten Voraussetzungen mittlerweile geschaffen, dass nun endlich wieder Ruhe (etwa in Gestalt des viel zitierten Schulfriedens) einkehrt im Lande ${ }^{8}$.

Menschen mit Behinderung selbst (oder im Falle von Kindern deren Eltern) kommen in diesem Prozess zwar eher zu Wort - den Möglichkeiten, ihre ambivalenten und nicht selten kritischen Erfahrungen mit dem Umsetzungsprozess aber wirksam zu Gehör verschaffen, sind nach wie vor jedoch enge Grenzen gesetzt. Überparteiliche und interfraktionelle Einigkeit im moralisch Gebotenen wirkt hier als neue Barriere, die einer Verschiebung von Repräsentationschancen in den Weg gestellt ist. Für akut Betroffene ändert sich zunächst wenig bis gar nichts. Hier und da kann der eine oder andere zusätzliche Integrationserfolg verzeichnet werden ${ }^{9}$ - aber von dem oft beschworenen Perspektivwechsel sind wir nach wie vor weit entfernt. Eine zukünftige Biografieforschung über in Zeiten der Inklusionsrhetorik aufgewachsene Menschen wird ergeben, dass diese Generation nur mit unerheblich weniger Teilhabebarrieren zu kämpfen hatte als die Generationen davor ${ }^{10}$. Die Provokation der UN-BRK droht im Strohfeuer von ,ein bisschen mehr Integration, soweit und sofern wir uns das in finanzpolitischen Krisenzeiten leisten können“", zu verpuffen.

8 So ein Originalkommentar auf dem Bildungskongress 2011 der CSU an der Hochschule Landshut.

9 So erhöhen die 41 in diesem Schuljahr in Bayern inthronisierten Schulen mit dem Schulprofil Inklusion zwar die Integrationsstatistik - gleichzeitig aber bleibt der Separationsanteil trotz insgesamt sinkender Schüler/innenzahlen tendenziell erstaunlich konstant. Integration und Separation werden so nicht zueinander ausschließenden Gegensätzen, sondern zu einer systemtheoretisch logischen Konsequenz eines fortgesetzt gewollten Nebeneinanders von Regel- und Sondereinrichtungen.

10 Vgl. hierzu Dorrance, Barrierefrei vom Kindergarten in die Schule? Eine Untersuchung zur Kontinuität von Integration aus der Sicht betroffener Eltern, Reihe Forschung, Bad Heilbrunn 2010; sowie Bruner, KörperSpuren. Zur Dekonstruktion von Körper und Behinderung in biografischen Erzählungen von Frauen, Bielefeld 2005. 


\section{Feuilletonistische Randbemerkungen aus dem beschädigten Leben oder (fast) wie im richtigen Leben}

\section{Die Grenzen der Inklusion in der inklusiven Praxis}

K. besucht eine Inklusionsschule. Er ist Inklusionsschüler. Seit Kurzem. I-Schüler war er schon immer. K. bereichert die Statistik. Mit und Dank ihm steigt die Inklusionsrate. Bei Problemen wendet er sich seit dem Schuljahr 2011/12 an seinen Inklusionslehrer. Der ist nunmehr zum Inklusionsbeauftragten anvanciert. Als solcher kann er stolz über die mittlerweile erreichte Inklusionsquote berichten. Das Soll ist erfüllt - natürlich stellt es eine Übertreibung dar, dass im kommenden Schuljahr eine Inklusionsecke der Begegnung im Pausenhof eingerichtet werden soll, die zu besuchen sich dann übrigens auch für Ali lohnen würde, dessen I-Bereitschaft ja bislang noch zu wünschen übrig lässt.

Tatsache ist aber, dass die Grenzen der Inklusion wohl irgendwo in oder vor Moskau liegen müssen. Man kennt ja diese heftigen Wintereinbrüche im Osten - das ist auch der Grund, weshalb K., dem I-Rollstuhlfahrer, eine Teilnahme an der gemeinschaftsbildenden Klassenfahrt in die Fremde leider auch in Inklusionszeiten nicht ermöglicht werden kann - oder haben Sie schon einmal einen Rollstuhl bei minus 20 Grad durch meterhohe Schneewehen geschoben (oder sich durch dieselben schieben lassen?) - das ist dem stärksten Bufti nicht zuzumuten und würde im Übrigen nun wirklich den allgemeinem Klassenfortschritt hemmen.

\section{Ramponierte Teilhabe - der vermessene inklusive Sozialraum}

„Für solche wie Euch haben WIR doch extra diese Rampen eingebaut“ flucht der MVG-Busfahrer, sich die öffentlichen Investitionen in den inklusiven Sozialraum vergegenwärtigend, als er bemerkt, dass sich der Rollstuhlfahrer K. mit seiner Begleitung unversehens und (womöglich auch) vorschriftswidrig des öffentlichen Raums selbstbestimmt, also unter Missachtung der zur Verfügung stehenden Rampe bemächtigt. Die Wege der Inklusion im Sozialraum sind schließlich vorgezeichnet und deutlich markiert - und die Teilhabe am Öffentlichen Nahverkehr führt in Deutschland nun mal vorzugsweise über Rampen. Schließlich dient das auch der Sicherheit der anderen Fahrgäste sowie der eigenen. Also: Die Normalen nehmen die normalen Eingänge. Der Rest benutzt die Resterampe. Noch stationenweit ist dem städtischen Busführer der Ärger über die augenscheinliche Fehlinvestition seiner Steuergelder deutlich ins Gesicht geschrieben. 


\section{Einsicht in die eigene Verstricktheit}

„Antithese - Für den, der nicht mitmacht, besteht die Gefahr, dass er sich für besser hält als die anderen und seine Kritik der Gesellschaft missbraucht als Ideologie für sein privates Interesse. Während er danach tastet, die eigene Existenz zum hinfälligen Bilde einer richtigen zu machen, sollte er dieser Hinfälligkeit eingedenk bleiben und wissen, wie wenig das Bild das richtige Leben ersetzt. Solchem Eingedenken aber widerstrebt die Schwerkraft des Bürgerlichen in ihm selber. Der Distanzierte bleibt verstrickt wie der Betriebsame, vor diesem hat er nichts voraus als die Einsicht in seine Verstricktheit und das Glück der winzigen Freiheit, die im Erkennen als solchem liegt.“11

\section{Inklusion einfach machen - und alles wird gut}

Bayern an der Spitze der Bewegung: „Inklusion durch Kooperation“ statt „Integration durch Kooperation" - Resultat eines Updates der ministeriellen Homepage ${ }^{12}$. Vollzugsmeldung: „München-Land ist schon inklusiv!“ - jüngst registrierte selbstbewusste Aussage von Seiten einer Schulamtsvertretung ${ }^{13}$. Vorsichtiger Optimismus: „München wird inklusiv!“ - Motto der Aktivitäten zur Erstellung eines städtischen Aktionsplans für die Landeshauptstadt ${ }^{14}$. Ein Gespenst geht um im Land: Wir werden jetzt mal schnell inklusiv. Alles wird gut.

\section{Wissenschaftlicher Teil}

Welche Konsequenzen ergeben sich aus einem inklusionsorientierten Blick auf den Sozialraum - bzw. aus einer sozialräumlichen Interpretation der Orientierung an Inklusion? Das Thema ist längst entdeckt: So lassen sich unschwer Veranstaltungsreihen finden, die sich mit „Sozialraum und Inklusion“ befassen ${ }^{15}$. Ebenso existieren Empfehlun-

11 Adorno, Minima Moralia. Reflexionen aus dem beschädigten Leben, Frankfurt a.M. 2001, S. 30 f.

12 Vgl. hierzu die Präsentation von MR Weigl vom Bayerischen Staatsministerium für Unterricht und Kultus: http://www.schulamt.mm.unterallgaeu.de/fileadmin/dateien/aktuelles/inklusion_durch_ kooperation_auftakt_folien_28_10_2009.pdf(Stand 10. Juni 2012).

13 Mündliche Aussage, getroffen auf dem Fachtag „Inklusion“ des Kreisjugendrings München-Land am 4. Mai 2012 im Rahmen des KJR-Jahresthemas 2012 und 2013 „Mädchen und Jungen mit Behinderung ins Blickfeld nehmen - Inklusion als Aufgabe für den KJR München-Land“.

14 Zum aktuellen Stand vgl. http://www.muenchen-wird-inklusiv.de/ (Stand: 10. Juni 2012).

15 Bspw. die gleichnamige Reihe, veranstaltet von der Europäischen Akademie für Inklusion: http://www.inklusion-sh.eu (Stand 10. Juni 2012). 
gen für den inklusiven Sozialraum, etwa des Deutschen Vereins für öffentliche und private Fürsorge e.V. ${ }^{16}$ Dort heißt es:

„I. Inklusion als gesellschaftliche Herausforderung

Wir leben in einer pluralistischen Gesellschaft, in der Menschen mit und ohne Behinderungen, alte und junge Menschen, mit oder ohne Migrationshintergrund gemeinsam Lebensräume nutzen und gestalten. Ein solidarisches Miteinander, in dem jede/r sein Leben individuell und selbstbestimmt in jeder Lebensphase - mal jung, mal alt, mal mit mehr Einschränkungen, mal mit weniger - gestalten kann, setzt gegenseitige Wertschätzung und die Erkenntnis voraus, dass sich jede/r gewinnbringend in die Gemeinschaft einbringen kann. Gelingt Inklusion nicht, sind Vernachlässigungen und Ausgrenzungen zu befürchten. Reparaturarbeiten zum Aufholen missglückter Inklusion sind aufwendig und ggf. kostspielig. Gelingt Inklusion, wird die Gesellschaft durch ihre Vielfalt bereichert, besteht weniger Anpassungsdruck für jede/ $\mathrm{n}$ und wird Solidarität gelebt. Eine inklusive Herangehensweise an Angebote des öffentlichen Raums insgesamt und des Sozialen im Besonderen vermindert Doppelarbeit, schafft Synergien und steigert damit die Effizienz der Angebote. Unterschiedliche Angebote für verschiedene Zielgruppen können durch eine inklusive Ausrichtung aller Angebote ggf. zusammengeführt werden. Auch ist davon auszugehen, dass in inklusiven barrierefreien Lebensräumen nicht alle, aber einige spezialisierte Angebote entbehrlich werden.

$[\ldots]^{“}$

Ich versuche den Sozialraum im Folgenden als einen Ort zu begreifen, an dem und in dem sich Inklusionsdiskurse manifestieren - handlungspraktisch, strukturell und rhetorisch. Insofern ist (vielleicht einzig) der Blick auf den Sozialraum geeignet, um eine diskurstheoretisch informierte Analyse von Inklusions- und Exklusionsverhältnissen durchzuführen und aufrecht zu erhalten. Deren Sinn bestünde darin, die Einlösung der normativen Forderung nach voller selbstbestimmter gesellschaftlicher Teilhabe, wie sie aus der UN-BRK abzuleiten ist, am Leben und Erleben aller im Sozialraum Agierenden zu messen.

Was wäre unter Inklusion zu verstehen, wenn aus ihr eine andere Praxis folgen soll als die, welche sich im Namen von Integrationsbemühungen für Menschen mit Behinderung und Menschen mit Migrationshintergrund in den letzten Jahrzehnten herausgebildet hat? Meine These: In diesem Sinne inklusive Entwicklungen, die den Namen verdienen, wären in dem Maße zu vermelden, in dem strukturelle Veränderungen zum Abbau vorhandener oder zur Verhinderung von neu errichteter Teilhabebarrieren führen und diese Prozesse von (institutionellen oder personellen) Entscheidungsträgern gestaltet und vollzogen werden, die ihr soziales Handeln einer anhaltenden Reflexion in Bezug auf die darin enthaltenen Inklusions- und Exklusionspotenziale unterziehen. Haben wir es mit informierten Inklusionshaltungen zu tun, die nicht in Strukturveränderungen eingebettet sind, so mag das wünschens- und allemal lobenswert sein (und auch manchem 'Betroffenen' vereinzelt zugutekommen), jedoch handelt es sich dabei lediglich

16 Vgl. hierzu http://www.deutscher-verein.de/05-empfehlungen/alteraltenhilfe/Eckpunkte_fuer_ einen_inklusiven_Sozialraum (Stand 10. Juni 2012). 
um Entwicklungen in einem integrationslogischen Kontext. Auch die umgekehrte Konstellation ist zu beobachten: Zusätzliche Ressourcen mögen zu verbesserten Rahmenbedingungen für den Umgang mit Vielfalt beitragen, allein die professionellen Einstellungen der Akteure, möglicherweise auch die Hartnäckigkeit wirksamer Vorurteile mögen einer Inklusionspraxis, die diesen Namen verdient, nach wie vor entgegenstehen. Inklusion so verstanden, beginnt nicht einerseits im Kopf und hängt andererseits vom politischen Willen ab - Inklusion bemisst sich vielmehr am Zustand der Gesellschaft insgesamt. Damit geraten einige Annahmen in den Fokus, die im Inklusionsdiskurs bisweilen zwar anklingen, selten aber in ihrer Konsequenz zu Ende gedacht werden:

Inklusive Prozesse erweisen sich nicht an einzelnen Best-Practice-Beispielen, Modellversuchen oder Leuchtturmprojekten - so begrüßenswert diese auch als exemplarische Belege für gelingende integrative Praxis sein mögen; diese entsprechen nicht dem, was unter einer Inklusionsorientierung zu verstehen wäre, nämlich der politische Wille, gesellschaftliche Teilsysteme in Bezug auf ihre Exklusionsdynamik fortgesetzt auf den Prüfstand zu stellen - im Sinne einer reflexiv begriffenen Gestaltungsaufgabe des Gemeinwesens in öffentlicher Verantwortung. Aus der Sicht so genannter Betroffener und das können wir bekanntlich alle sein, in je unterschiedlichen biografischen Konstellationen des Lebenslaufs - ist das Erleben des barrierefreien und Teilhabe ermöglichenden Sozialraums der Maßstab für Inklusionsqualität - und nicht etwa das eventuelle Vorhandensein einer Schwerpunktschule mit dem Schulprofil Inklusion oder ähnliches. Die Kategorie der Behinderung ebenso wie die der Barrierefreiheit verliert dabei ihre Tauglichkeit zur Unterscheidung von Zielgruppen. Sozialraumbezogene Inklusionsprozesse manifestieren sich demnach nicht in einer (oft notwendigen und wünschenswerten) Statusverbesserung Benachteiligter oder Diskriminierter, sondern sie manifestieren sich im Abbau und in der Verhinderung von Diskreditierungs- und Diskriminierungspotenzialen, die mit den gesellschaftlich bedeutsamen bzw. bedeutsam gemachten Differenzen wie Gender, Herkunft, Alter, sexuelle Orientierung, kultureller Identität oder den ökonomischen Verwertungspotenzialen individuellen Leistungsvermögens verknüpft werden. Insofern verschiebt sich im Rahmen von sozialraumorientierten Inklusionsbemühungen gewissermaßen der Fokus des Blicks - sozialraumorientierte Inklusionsbemühungen erweisen sich somit nicht in der Etablierung zusätzlicher Pilotprojekte, in denen die Bedingungen eines „gelingenden Umgangs mit Vielfalt“ erprobt werden, sondern in Praxen, die sich theoretisch an intersektionalen Fragestellungen orientieren - z.B. in einer Querschnittsanalyse der Wirksamkeit rassistischer Logiken im Sozialraum, auch innerhalb eines Diskurskontextes, der durch positive Konnotationen von kultureller Differenz gekennzeichnet ist. Insbesondere Mecheril 17 weist in diesem Zusammenhang darauf hin, dass dies etwa auch zur Verfestigung der Annahme von statischen Kulturen führen kann. Insofern sind kulturanthropologische Annahmen kultureller Differenz und die soziale Wirksamkeit kultureller Prägungen

17 Mecheril, Migrationspädagogik, Weinheim, Basel, Berlin 2010. 
stets aufeinander und miteinander bezogen. Ein weiteres Beispiel sozialraumorientierter Inklusionsbemühungen würde die institutionalisierte Bearbeitung der Frage nach sozioökonomischen Ungleichheitsentwicklungen darstellen, die sich im Sozialraum abbilden und die Bedingungen des Zusammenlebens bestimmen. Dabei geht es im Rahmen einer intersektionalen Perspektive stets um die Frage der Wechselwirkungen zwischen den gesellschaftliche Bedeutsamkeit erlangenden Differenzmerkmalen.

Es ist jeweils die unauflösbare Ambivalenz des Spannungsfelds von Vielfalt und Differenz, das die zentrale Bedingung für sozialraumorientierte Inklusionsbemühungen darstellt. Eine einseitige Auflösung dieser Ambivalenz zugunsten der Anerkennung von Vielfalt führt zur folkloristischen Überhöhung des „Alles so schön bunt hier“, die blind für die reale Wirksamkeit normierender Machtverhältnisse oftmals in einer Bereicherungsrhetorik verharrt - die Auflösung der unhintergehbaren Ambivalenz zugunsten einer Fokussierung auf die Kritik von Differenzverhältnissen übersieht hingegen nicht selten die realexistierende Vielfalt vom Blickwinkel einer verallgemeinernden Identitätsposition aus, die vor allem den der eigenen Position inhärenten hegemonialen Deutungsanspruch nicht kritisch zu reflektieren vermag.

Bereits bei Prengel 18 deutete sich an, dass im pädagogischen Diskurs mit Blick auf Geschlecht, Kultur und Behinderung jeweils ähnliche Verlaufsmuster der Betrachtung von Vielfalt $\mathrm{zu}$ beobachten waren und sind. Man könnte die Genese der fachlichen Blickrichtungen davon ausgehend als eine Entwicklung von Defizit- über Differenz- zu Bereicherungsparadigmen beschreiben, wobei sich die Diskurse nicht jeweils notwendigerweise parallel und in gleicher Intensität und Geschwindigkeit entwickelten. Insbesondere im heutigen handlungspraktisch ausgerichteten pädagogischen Inklusionsdiskurs, der sich auf die Konsequenzen beruft, die sich aus der UN-BRK ergeben, scheint das Bereicherungsparadigma erst punktuell kritisch hinterfragt ${ }^{19}$. Eine Position der Kritik ist jedoch - blickt man auf die benachbarten Gender- und Kulturdiskurse - in dem Maße zu erwarten, in dem Menschen mit Behinderung selbst sich zunehmend in der Lage sehen, ihre Repräsentationspotenziale zu nutzen. Das allerdings hätte zur Folge, dass sich die Definitionsmacht über Form, Inhalt und Gestalt von zukünftigen Inklusionsprozessen gewissermaßen selbst vervielfältigt - und ggf. mancher selbsternannten Kampfinstanz für Inklusion unversehens zwischen den Fingern zerrinnt.

Ein - außerhalb Bayerns - erprobtes und verbreitetes Selbstevaluationsinstrument zur Gestaltung kommunaler Inklusionsentwicklung ist der kommunale Index für Inklusion. Es handelt sich um eine Übersetzung und Adaption des von Booth und Ainscow

18 Vgl. Prengel, Pädagogik der Vielfalt. Verschiedenheit und Gleichberechtigung in Interkultureller, Feministischer und Integrativer Pädagogik. Schule und Gesellschaft, Bd. 2, Wiesbaden 2006.

19 Vgl. dazu bspw. Rathgeb, Disability Studies. Die Perspektive beeinträchtigter Menschen. Reihe: Perspektiven kritischer Sozialer Arbeit, Wiesbaden 2012; sowie Anhorn u.a., Kritik der Sozialen Arbeit - kritische Soziale Arbeit. Perspektiven kritischer Sozialer Arbeit, Wiesbaden 2012. 
$(2002)^{20}$ zuerst entwickelten Index for Inclusion ${ }^{21}$, der mittlerweile neben der deutschen Übersetzung von Boban/Hinz ${ }^{22}$ auch in einer speziellen Fassung für Kindertageseinrichtungen ${ }^{23}$ vorliegt. Im kommunalen Index für Inklusion geht es um „Diversität in der Entwicklung eines inklusiven Gemeinwesens" 24 . Kommune wird dabei - im tendenziellen Unterschied zu Ansätzen kommunaler Teilhabeplanung25, die auch explizit auf Menschen mit Behinderung zielen - nicht nur als lokale Verwaltungseinheit begriffen, sondern als

„große Gemeinschaft: In ihr leben Menschen zusammen, in vielen Formen und auf vielen Ebenen. Hier können Menschen im Austausch mit anderen Menschen und der Verwaltungsebene ihres Ortes gemeinsam wirksam werden" 26 .

Diese an Vielfalt orientierte Perspektive schließt folgende Aktivitätsebenen der prozesshaften Inklusionsentwicklung ein:

- die Kommune als Wohn- und Lebensort;

- die inklusive Entwicklung kommunaler Organisationen;

- die Kooperation und Vernetzung innerhalb der Kommune.

Die Arbeit mit dem Kommunalen Index für Inklusion verspricht:

- Verbesserungspotenziale zu identifizieren und realisierbare Schritte zeitnah umzusetzen;

- durch organisationsübergreifende Aktivitäten auf kommunaler Ebene Synergieeffekte zu erreichen;

- das gesellschaftliche Bewusstsein zur Wertschätzung von Unterschiedlichkeit zu erhöhen;

20 Booth/Ainscow, Index for Inclusion, Developing Learning and Participation in Schools. Center for Studies on Inclusive Education, 3. Aufl. London 2011.

21 Montag Stiftung Jugend und Gesellschaft (Hrsg.), Kommunaler Index für Inklusion. Arbeitsbuch. Montag Stiftung, Köln 2010, abrufbar unter: http://www.montagstiftungen.com/fileadmin/

Redaktion/Jugend_und_Gesellschaft/PDF/Projekte/Kommunaler_Index/KommunenundInklusion Arbeitsbuch_web.pdf (- Stand 10. Juni 2012).

22 Boban/Hinz, Index für Inklusion - Lernen und Teilhabe in der Schule der Vielfalt entwickeln, Halle 2003, http://www.eenet.org.uk/resources/docs/Index\%20German.pdf (Stand 10. Juni 2012).

23 Gewerkschaft Erziehung und Wissenschaft (GEW), Index für Inklusion (Tageseinrichtungen für Kinder. „Lernen, Partizipation und Spiel in der inklusiven Kindertageseinrichtung entwickeln“. Deutschsprachige Ausgabe. http://www.fhoow.de/sowe//downloads/12/index_veranstaltung _ emden.pdf (Stand 10. Juni 2012).

24 Lawrenz, Diversität in der Entwicklung eines inklusiven Gemeinwesens: Erfahrungen mit dem „Kommunalen Index für Inklusion“, Archiv für Wissenschaft und Praxis der Sozialen Arbeit. Diversity Management und soziale Arbeit 1/2012, S. 83-91.

25 Vgl. hierzu etwa die Arbeiten von Rohrmann, http://www.buergergesellschaft.de/fileadmin/pdf/ gastbeitrag_rohrmann_100430.pdf (Stand 10. Juni 2012) und Lampke/Rohrmann/Schädler, Örtliche Teilhabeplanung mit und für Menschen mit Behinderungen: Theorie und Praxis, Wiesbaden 2011.

26 Imhäuser, in: Montag Stiftung Jugend und Gesellschaft (Hrsg.), Inklusion vor Ort - Der Kommunale Index für Inklusion - Ein Praxishandbuch, Berlin 2011, S. 8. 
- Selbstwirksamkeit im direkten Lebensumfeld zu erfahren und zu gestalten;

- barrierefreie Beteiligung an der Gestaltung des kommunalen Zusammenlebens und -wirkens zu verbessern;

- Dienstleistungen kommunaler und anderer Träger an Bedürfnis und Bedarf der Bevölkerung anzupassen.

Ansatzpunkt für die Initiierung, Kontrolle und kontinuierliche Fortentwicklung des Inklusionsprozesses ist dabei der einzelne handelnde Akteur. Ihre verändernde Wirkung entfalten fünf voneinander unterscheidbare Ebenen der Kommune primär über die auf gutem Willen, Motivation und „richtiger“ Einstellungen basierende Praxis handelnder Subjekte. „Andere Menschen mit der Bedeutung von Inklusion 'infizieren', die Idee weitertragen und weitere Aktive und Multiplikator/-innen gewinnen, ist ein zentraler Aspekt von inklusiven Prozessen“27. Die inklusive Entwicklung nimmt dabei ihren Ausgangspunkt in einer reflexiven Versicherung eines angemessenen Inklusionsverständnisses auf personaler/individueller Ebene, markiert dann den Sozialraum (begriffen als „Nachbarschaftsebene“) in Gestalt alltäglicher Begegnungen und setzt sich dann fort auf der Ebene von Organisationen, deren Vernetzung bis hin zur kommunalen/politischen Ebene, die im Sinne einer ,großen inklusiven Gemeinschaft" beschrieben wird. Kern inklusiver Prozesse ist dabei Partizipation: „Inklusion als Schlüssel für eine zukunftsfähige Gesellschaft kann nur in partizipativen Prozessen erreicht werden, in denen die Menschen mitgestalten, ihre Selbstwirksamkeit erfahren und Verantwortung übernehmen können“'28.

\section{Ausblick}

Diesem ohne Zweifel praxisorientierten und praxisbewährten Ansatz zu Initiierung, Aufrechterhaltung und Fortentwicklung integrativer Prozesse wohnt ebenso unübersehbar ein normativer und moralischer Impetus inne, der - so meine These - geeignet ist, vom kritischen Potenzial der Inklusionsforderung, wie sie in der UN-BRK aufscheint, eher abzulenken. Der Ansatz, Exklusions- und Inklusionsverhältnisse im Sozialraum quasi von unten strukturell verändern zu wollen, ohne diese Verhältnisse gleichzeitig einer strukturellen Analyse und reflexiven Kritik zu unterziehen, muss zwangsläufig und bestenfalls in Leuchtturmprojekten enden, was durchaus auch zu einer punktuell vorbildhaften ,inklusiven Gemeinwesenentwicklung“ 29 beitragen kann. Der nachhaltige Erfolg dieser Impulse bleibt jedoch abhängig von der geteilten Motivationslage und der

27 Lawrenz (Fußn. 24).

28 Lawrenz (Fußn. 24).

29 Vgl. hierzu etwa die inklusiven Entwicklungen in der Gemeinde Wiener Neudorf, dokumentiert auf: http://www.wiener-neudorf.gv.at/system/web/zusatzseite.aspx?menuonr=218605890\&detailonr= 218608690 (Stand 10. Juni 2012). 
Verständigung darüber, wie Vielfalt und deren anerkennende Wahrnehmung jeweils begrifflich gefasst werden. Da eine systematische Analyse der gesellschaftlichen Verhältnisse, auf denen Integrationsprozesse beruhen, nicht vorgesehen ist, erscheinen diese methodischen Ansätze auch wenig gegen politische Umarmungs- und Instrumentalisierungsstrategien gewappnet zu sein. „Ob Inklusion nachhaltig umgesetzt werden kann, wird sich maßgeblich darin zeigen, inwieweit sich die Wertschätzung von Vielfalt und die Umsetzung inklusiver Werte im täglichen Miteinander widerspiegeln“30. Möglicherweise stellt die in der Neuausgabe des Index for Inclusion ${ }^{31}$ erfolgte Weiterentwicklung des methodischen Ansatzes eine Konsequenz aus seiner theoretischen Unterkomplexität dar. Hier scheinen über die kommunikativ-handlungspraktisch begriffenen „,inklusiven Werte“ hinaus Aspekte in die Betrachtung einbezogen, die Anknüpfungspunkte für gesellschaftskritische Perspektiven bieten könnten.

Die transdisziplinäre Herausforderung für eine wissenschaftliche Befassung mit dem Umsetzungsprozess der UN-BRK liegt darüber hinaus in der Analyse der Strukturen des herrschenden Inklusionsdiskurses und der nicht zuletzt auch in ihm enthaltenen und sich spiegelnden Machtverhältnisse. Wer spricht warum und in welcher Absicht von Inklusion (und nicht von Integration) - und mit welchen Folgen? Wie verändert der herrschende Inklusionsdiskurs die Wahrnehmungen von Vielfalt, die Herstellungspraxen von Differenz(en)? Für welche Diskurse erweist sich der Inklusionsdiskurs als anschlussfähig und wo bzw. wie werden diese Anschlüsse hergestellt (und wo nicht)? Welche Entwicklungen werden angestoßen, wenn etwa die Debatte um Interkulturelle Öffnung sozialer Einrichtungen sich mit der „benachbarten“ Diskussion um ein inklusives Bildungssystem zu verständigen sucht? Welches Schicksal erfahren hierarchisierte Differenzen in der Gesellschaft angesichts von Diversityorientierung und Mainstreamingstrategien? Welche Effekte hat die „Entdeckung von Vielfalt“ in und durch eine Pädagogik, die sich selbst als inklusiv begreift? Und zu guter Letzt: Verlieren dabei Behinderung, kulturelle Herkunft, Genderzugehörigkeit, sexuelle Orientierung oder Alter etwas von ihren Exklusionspotenzialen, ihrer Diskreditierbarkeit?

Vielleicht ist aber Inklusion schlussendlich eben auch keine „Lösung“ - zumindest nicht der Beleg für ein Stück Richtiges im Falschen - sondern lediglich ein weiterer Begründungzusammenhang für den Auftrag zur Gestaltung einer menschengerechteren Welt und der Notwendigkeit, sich mit den gegebenen Verhältnissen nicht zufriedenzugeben.

30 Lawrenz (Fußn. 24)

31 Vgl. auch Booth, Wie sollen wir zusammen leben? Inklusion als wertbezogener Rahmen für die pädagogische Praxis, in: Gewerkschaft Erziehung und Wissenschaft (Hrsg.), Jugendhilfe und Sozialarbeit, 2. Auflage Frankfurt a.M. 2011. 
